\title{
Treatment Quality through Advanced Practice Nurses (APN) and Clinical Nurses (CN) in Ambulatory Primary Health Care - a retrospective study Behandlungsqualität durch Advanced Practice Nurses (APN) und Clinical Nurses (CN) in der ambulanten Gesundheitsversorgung - eine retrospektive Studie
}

Christian Ambrosch ${ }^{1 *}$, Isabelle Mathier ${ }^{1}$, Irina Bajusic ${ }^{1}$, Thomas Bucher ${ }^{2}$, Chantal Wüst ${ }^{1}$

\author{
${ }^{1}$ Gemeinschaftspraxis Medase Winterthur Neuwiesen, \\ 8400 Winterthur, Schweiz \\ *christian.ambrosch@medbase.ch \\ ${ }^{2} Z$ ürcher Hochschule für Angewandte Wissenschaften \\ ZHAW, Departement Gesundheit, 8401 Winterthur, Schweiz
}

Received 24 September 2020, accepted 6 October 2020

\begin{abstract}
Introduction: The shortage of general practitioners in Switzerland requires new ways to ensure primary outpatient health care. The group practice Medbase Winterthur Neuwiesen has been testing the use of Advanced Practice Nurses (APN) and Clinical Nurses (CN) for the treatment of clearly defined cases with minor illnesses for the past two years. In the context of quality assurance, this study examines the quality of treatment and outcome in 60 patients with symptoms of an acute upper respiratory tract infection, half of whom were assigned to a physician and half to an APN/CN

Methods: Analysis of the treatment and the outcome of 60 patient files, half of which were randomly assigned to a physician and half to an APN/CN.

Results: APN/ CN treated significantly more often according to standardized guidelines than physicians; the outcome in patients treated by APN/ $\mathrm{CN}$ was slightly better, the difference is not significant, though.

Discussion: The use of APN and CN in outpatient primary care is possible without compromising the quality of treatment in cases with minor illnesses and clearly defined guidelines for treatment.
\end{abstract}

\begin{abstract}
Einleitung: Der Mangel an Hausärzten erfordert auch in der Schweiz neue Wege, um die ambulante Grundversorgung sicherzustellen. Die Gruppenpraxis Medbase Winterthur Neuwiesen erprobt seit 2 Jahren den Einsatz von Advanced Practice Nurses (APN), resp. Clinical Nurses (CN) für die Behandlung klar definierter Fälle mit leichten Erkrankungen. Im Rahmen der Qualitätssicherung untersucht diese Studie die Behandlungsqualität und den Outcome bei 60 Patienten/-innen mit Symptomen eines akuten Infektes der oberen Atemwege, die je zur Hälfte einer Ärztin/einem Arzt resp. einer APN/CN zugeteilt wurden

Methode: Retrospektive Analyse der Behandlung und deren Outcome anhand von 60 Patientenakten, die je zur Hälfte zufällig einer Ärztin/ einem Arzt resp. einer APN/CN zugeteilt wurden.

Resultate: APN/CN behandelten signifikant häufiger gemäß standardisierten Leitlinien als Ärzte/-innen; der Outcome war bei von KMT behandelten Patienten/innen etwas besser, der Unterschied ist jedoch nicht signifikant.

Diskussion: Der Einsatz von APN und CN in der ambulanten hausärztlichen Versorgung ist möglich ohne Qualitätseinbuße bei der Behandlung von definierten Krankheitsbildern und klaren Leitlinien für deren Behandlung.
\end{abstract}

\section{Keywords}

clinical nurse - advanced practice nurse - physician assistants/associates - treatment quality - Outcome - outpatient care

\section{Keywords}

klinischer Fachspezialist - advanced practice nurse - physician assistants/associates - Behandlungsqualität - Outcome - ambulante Versorgung 


\section{EINLEITUNG}

\section{Hintergrund}

Die ambulante Gesundheitsversorgung in der Schweiz zeichnet sich durch einen zunehmenden Mangel an Hausärzten/innen aus. Das Bundesamt für Gesundheit (BAG)hatdieseSituationerkanntund siehtinderFörderung der Interprofessionalität einen möglichen Lösungsweg (BAG, 2017). Im entsprechenden Förderprogramm „Interprofessionalität im Gesundheitswesen“ heißt es: „Die medizinische Versorgung in der Schweiz steht vor großen Herausforderungen. Zum einen führen die demografische Alterung sowie die Zunahme an chronisch erkrankten Personen zu einem wachsenden Bedarf an medizinischen Leistungen. Zum anderen ist es bereits heute schwierig, ausreichend qualifiziertes (...) Personal im Gesundheitswesen zu rekrutieren. Diese Situation wird sich aus demografischen Gründen in den kommenden Jahren nicht ohne Weiteres entspannen. Interprofessionelle Zusammenarbeit stellt einen Ansatz dar, mit dem diesen Herausforderungen begegnet werden kann“" (ibid. S. 5).

Die interprofessionelle Zusammenarbeit kann durch den Einsatz von nicht-ärztlichem medizinischen Personal gestaltet werden, was in der direkten Patientenversorgung in vielen Ländern wie Großbritannien, USA, Kanada, Australien, Holland und Finnland bereits seit Jahrzehnten fest im Gesundheitssystem sowie in der Primärversorgung etabliert ist (Maier et al., 2017).

\section{Advanced Practice Nurse, Clinical Nurse: neue Rollen in der Gesundheitsversorgung}

Es handelt sich bei dieser Berufsgruppe um diplomierte Pflegefachpersonen, welche durch ein Studium medizinische Kenntnisse vertiefen und spezifische Fähigkeiten für die Untersuchung und Behandlung von Patienten/-innen erwerben. Diese Advanced Practice
Nurses (APN) und Clinical Nurses (CN) führen nach abgeschlossener Ausbildung medizinische Tätigkeiten in einem definierten Tätigkeitsfeld selbständig und unter ärztlichen Supervision durch. Sie arbeiten im Team/Tandem mit dem ärztlichen Dienst und weiteren Gesundheitsberufen zusammen. Sie bilden somit neben den klassischen Rollen in der Primärversorgung mit Ärzten/-innen auf der einen und medizinischen Praxisassistenten/-innen (MPA) auf der anderen Seite ein drittes Rollenbild. Ein einheitlicher Begriff konnte sich für dieses Rollenbild in der Schweiz bisher nicht durchsetzen. In den USA hat sich für diese Berufsgruppe die Sammelbezeichnung „Advanced Practice Nurse“ etabliert. Andere Begriffe werden dafür ebenfalls verwendet: Clinical Nurse, Nurse Practionier, Physician Assistant oder Physician Associtate.

In der Schweiz bestehen derzeit zwei Bildungsabschlüsse, um diese Funktionen ausführen zu können (vgl. Tabelle 1). Die Funktion APN setzt einen Master-Abschluss in Pflegewissenschaft an einer dafür akkreditierten Universität oder Hochschule voraus. Weiter besteht die Möglichkeit, als Weiterbildung ein CAS-Diplom zu erwerben als klinische Fachspezialistin/klinischer Fachspezialist (KFA), resp. Clinical Nurse.

Der Einsatz von APN und CN wird auch in der Schweiz in der stationären Versorgung bereits erprobt, deren Regulierung ist jedoch ausstehend wie die Beiträge an den APN-Symposien der Zürcher Hochschule für Angewandte Wissenschaften ZHAW zeigen (ZHAW, 2020). Für die ambulante Versorgung wurden ebenfalls Einsatzgebiete diskutiert und erprobt, zum Beispiel bei der Versorgung chronisch kranker Patienten/-innen und bei Hausbesuchen (vgl. Konferenz der kantonalen Gesundheitsdirektorinnen und -direktoren, 2019). Diese Funktionen sind in der hausärztlichen Grundversorgung jedoch noch nicht etabliert.

Tabelle 1: Rollenbilder und Funktionen von APN und CN in der Schweiz, eigene Darstellung

\begin{tabular}{|c|c|c|}
\hline Funktionsbezeichnung & Advanced Practice Nurse (APN) & Clinical Nurse (CN) \\
\hline $\begin{array}{l}\text { alternative/ähnliche } \\
\text { Funktionsbezeichnungen }\end{array}$ & $\begin{array}{l}\text { - Pflegeexpertin/Pflegeexperte APN } \\
\text { - Nurse Practitioner } \\
\text { - Clinical Nurse Specialist }\end{array}$ & $\begin{array}{l}\text { - Klinische Fachspezialistin/Klinischer Fachspezialist } \\
\text { (KFA) } \\
\text { - Physician Assistant/Physician Associate }\end{array}$ \\
\hline Ausbildung & $\begin{array}{c}\text { für die Funktionsbezeichnung APN benötigt eine } \\
\text { diplomierte Pflegefachperson FH einen Abschluss } \\
\text { Master of Science in Nursing mit klinischer } \\
\text { Vertiefung }\end{array}$ & $\begin{array}{l}\text { für die Funktionsbezeichnung KFA benötigt } \\
\text { eine diplomierte Pflegefachperson HF/FH einen } \\
\text { Abschluss CAS Klinische Fachspezialisten }\end{array}$ \\
\hline $\begin{array}{l}\text { Hauptunterschiede } \\
\text { Aufgabengebiete }\end{array}$ & $\begin{array}{c}\text { - mehr Eigenverantwortung } \\
\text { - mehr Entscheidungskompetenz } \\
\text { - Betreuung von komplexeren und multimorbiden } \\
\text { Patienten } \\
\text { - Leadership und Ausbildung } \\
\text { - Forschung }\end{array}$ & $\begin{array}{c}\text { - unkomplizierte Behandlungsfälle } \\
\text { - standardisierte Untersuchungen (ABI-Messung/ } \\
\text { Schellong-Test usw.) } \\
\text { - vermehrt Instruktionen (auch an MPA) } \\
\text { - vermehrt Tätigkeit unter ärztlicher Supervision }\end{array}$ \\
\hline
\end{tabular}




\section{Ziel der Studie}

In der Gruppenpraxis Medbase Winterthur Neuwiesen kommen seit rund zwei Jahren in der ambulanten Versorgung neben Allgemeinmedizinern und MPA auch APN und $\mathrm{CN}$ zum Einsatz. Zwei $\mathrm{CN}$ und eine APN (cand.) sind im medizinischen Bereich tätig und gut im Arbeitsalltag integriert. Da die beiden Funktionen APN und $\mathrm{CN}$ nicht einheitlich verwendet werden können, benutzt die Gruppenpraxis Medbase zur Vereinfachung intern den Begriff „Klinisch-Medizinisches Team“ (KMT). Das KMT übernimmt nebst pflegerischen Aufgaben klar definierte ärztliche Tätigkeiten unter ärztlicher Supervision. Um die ersten positiven Erfahrungen mit Evidenz zu untermauern, führte die Gruppenpraxis eine Evaluation der Behandlungsqualität bei Patienten/-innen mit Symptomen eines akuten Infektes der oberen Atemwege durch. Dafür wurde die Qualität und der Outcome von Behandlungen verglichen, die das KMT durchführte mit solchen, die Ärzte/-innen vornahmen. Die Forschungsfrage für diesen Beitrag lautet damit: Unterscheidet sich die Behandlung von Patienten/-innen mit Atemwegsinfekten und deren Outcome abhängig von der Behandlung durch ein Ärzteteam resp. durch das KMT?

\section{METHODEN}

\section{Setting und Auswahl der Fälle}

Das KMT ist ein Teil der hausärztlichen Grundversorgung der Gruppenpraxis Medbase Winterthur Neuwiesen, in der insgesamt 13 Allgemeinmediziner/-innen arbeiten. Vom KMT arbeiten zwei CN und eine cand. APN in der Allgemeinmedizin. Sie haben eine eigene SprechstundenAgenda, in der nach vorheriger Telefontriage durch telemedizinisch geschultes Praxispersonal als unkompliziert eingestufte Behandlungsfälle terminiert werden. $\mathrm{Zu}$ diesem Spektrum gehören auch Patienten/innen mit Symptomen eines akuten Infektes der oberen Atemwege. Je nach Kapazität werden diese einem Arzt/ einer Ärztin oder dem KMT zugeteilt. Da die Zuteilung nach jeweiliger Kapazität in der Sprechstunde erfolgte, besteht grundsätzlich eine Randomisierung. Patienten/innen, bei denen die Diagnose einer Pneumonie gestellt wurde, wurden von der Analyse ausgeschlossen. Die Ärzte/-innen haben eine Konsultationszeit bei Atemwegsinfekten von $15 \mathrm{~min}$. Dem KMT stehen $30 \mathrm{~min}$. zur Verfügung. Anhand von einem für die Praxis, durch einen auch als Lehrarzt tätigen Allgemeinmediziner, mit dem KMT erarbeiteten Handbuch für häufige Behandlungsfälle, erfolgte die Entscheidungsfindung des KMT. In dem Handbuch sind die aktuellen evidenzbasierten Leitlinien der Schweizerischen Gesellschaft für Infektiologie (SGINF, 2020) und klare Behandlungspfade integriert. Die Behandlung einer unkomplizierten (meist viralen) Infektion der oberen Atemwege sieht demnach in den meisten Fällen keinen Einsatz von Antibiotika vor, sondern Maßnahmen zur Symptombekämpfung wie zum Beispiel fiebersenkende Schmerzmittel (NSAR, Paracetamol), Hustenmittel und Sekretolytika. Bei Unsicherheit steht ein definierter ärztlicher Supervisor für Fragen dem KMT zur Verfügung. Ansonsten erfolgte die Supervision der Behandlung im Laufe des Tages anhand des Behandlungseintrages in der elektronischen Patientenakte durch den Supervisor.

Vor der Konsultation erhält der Patient/die Patientin als Praxisstandard bei Infekt-Beschwerden immer eine kapilläre Blutabnahme mit Bestimmung der Laborparameter CRP und kleinem Blutbild.

Für die Evaluation wurden die elektronischen Akten von Patienten/-innen mit unkomplizierten Infekten der oberen Atemwege aus dem Zeitraum Januar bis Februar 2020 vor dem Auftreten der Corona-Pandemie ausgewählt. In den Wintermonaten ist die Inzidenz von Atemwegsinfekten bekanntlich hoch. Verglichen wurden je 30 Behandlungen, die von insgesamt neun Allgemeinmediziner/-innen (Ärzte-Gruppe), resp. dem KMT durchgeführt wurden (KMT-Gruppe).

\section{Operationalisierung der Behandlungsqualität und des Outcomes}

Die Behandlungsqualität wurde daran gemessen, ob diese den aktuellen Empfehlungen zur Antibiotikaverschreibung bei unkomplizierten Atemwegsinfekten entsprach: Symptombekämpfung und keine Antibiotikaverschreibung.

DerOutcome wurde anhand des Verlaufs der Symptomatik gemessen. Da Patienten/-innen sich bei fehlender Besserung üblicherweise erneut in der Praxis vorstellen oder die Praxis einen Brief über eine Behandlung in einer Notfallambulanz erhält, wurde als positives Outcome gewertet, wenn die Patienten/-innen bei einer geplanten Nachkontrolle mit deutlicher Besserung vorstellig wurden oder wegen dieses Erkrankungsfalles weder in unserer Praxis noch in einer Notfallpraxis erneut behandelt werden mussten.

\section{Datenanalyse}

Die Behandlungsqualität und der Outcome wurden mittels Vierfeldertafel und dem Chi-Quadrat-Test mit Kontinuitätskorrektur verglichen. Die Analyse erfolgte mit dem Statistikprogramm SPSS Version 26 von IBM. 


\section{ERGEBNISSE}

\section{Charakteristika der Patienten/-innen}

Die Charakteristika der Patienten/-innen zeigt Tabelle 2:

Tabelle 2: Charakteristik der Patienten/-innen.

\begin{tabular}{c|c|c}
\hline Patientencharakteristik & $\begin{array}{c}\text { Ärte-Gruppe } \\
\mathbf{N = 3 0}\end{array}$ & $\begin{array}{c}\text { KMT-Gruppe } \\
\mathbf{N}=\mathbf{3 0}\end{array}$ \\
\hline Altersdurchschnitt & $39 \mathrm{~J}$ & $31 \mathrm{~J}$ \\
\hline $\begin{array}{c}\text { Geschlecht } \\
\text { - männlich } \\
\text {-weiblich }\end{array}$ & $\begin{array}{c}43 \%(\mathrm{~N}=13) \\
57 \%(\mathrm{~N}=17)\end{array}$ & $\begin{array}{c}63 \%(\mathrm{~N}=19) \\
37 \%(\mathrm{~N}=11)\end{array}$ \\
\hline Relevante Vorerkrankung & $37 \%(\mathrm{~N}=11)$ & $27 \%(\mathrm{~N}=8)$ \\
\hline Temperaturdurchschnitt in ${ }^{\circ} \mathrm{C}$ & 37 & 37 \\
\hline BD/P gemessen in & $20 \%(\mathrm{~N}=6)$ & $97 \%(\mathrm{~N}=29)$ \\
\hline $\begin{array}{c}\text { Laborparameter } \\
\text { CRP-Durchschnitt in } \mathrm{mg} / \mathrm{L}\end{array}$ & 22 & 18 \\
\hline Neutrophile-Durchschnitt in $\mathrm{mm}{ }^{3} / \mathrm{L}$ & 6 & 5 \\
\hline Leukozyten-Durchschnitte in $10^{3} / \mathrm{uL}$ & 9 & 7 \\
\hline $\begin{array}{c}\text { Diagnosen } \\
\text { Unspezifische Infekte der } \\
\text { Atemwege }\end{array}$ & $64 \%(\mathrm{~N}=19)$ & $47 \%(\mathrm{~N}=14)$ \\
\hline Pharyngitis & $3 \%(\mathrm{~N}=1)$ & $20 \%(\mathrm{~N}=6)$ \\
\hline Sinusitis in & $3 \%(\mathrm{~N}=1)$ & $10 \%(\mathrm{~N}=3)$ \\
\hline Bronchitis in & $10 \%(\mathrm{~N}=3)$ & $10 \%(\mathrm{~N}=3)$ \\
\hline Angina tonsillaris in & $10 \%(\mathrm{~N}=3)$ & $10 \%(\mathrm{~N}=3)$ \\
\hline Otitis media in $\%$ & $10 \%(\mathrm{~N}=3)$ & $3 \%(\mathrm{~N}=1)$ \\
\hline
\end{tabular}

Das Durchschnittsalter in der KMT-Gruppe lag bei 31 Jahren im Vergleich zu 39 Jahren in der Ärzte-Gruppe. Die Geschlechtsverteilung zeigte einen größeren Anteil von Männern (63\%) in der KMT-Gruppe, wohingegen in der Ärzte-Gruppe mehr Frauen (57\%) behandelt wurden. In der KMT-Gruppe wiesen $27 \%$ eine relevante Vorerkrankung (Herz-Kreislauf-Erkrankung, Diabetes, Lungenerkrankung, aktives Tumorleiden, Immunsuppression) auf gegenüber $37 \%$ in der Ärzte-Gruppe.

In der Laboranalyse lag der durchschnittliche CRPWert mit $18,3 \mathrm{mg} / \mathrm{l}$ in der KMT-Gruppe etwas niedriger als in der Ärzte-Gruppe von $22,3 \mathrm{mg} / 1$, die Leukozyten befanden sich im Schnitt bei 8,6 × 109/1 (Neutrophile $\left.5,8 \times 10^{9} / 1\right)$ bei der Ärzte-Gruppe im Vergleich zu $7,1 \times 10^{9} / 1$ (Neutrophile 4,7 × 109/1) in der KMT-Gruppe. Die Vitalwerte (Blutdruck, Herzfrequenz, Körpertemperatur) wurden in der KMT-Gruppe bei 29 Patienten/-innen $(97 \%)$ und damit deutlich häufiger bestimmt. Bei den Ärzten wurde bei 9 Patienten die Temperatur und bei 6 Patienten Blutdruck und Herzfrequenz gemessen.

Die mit Abstand häufigste dokumentierte Diagnose war die eines unspezifischen Infektes der Atemwege mit den typischen Symptomen Husten, Schnupfen, Halsschmerzen (64\% bei den Ärzten vs. $47 \%$ beim KMT). Bei den spezifischen Diagnosen wurde bei der KMT-Gruppe eine Pharyngitis am häufigsten gestellt ( $20 \%$ vs. $3 \%$ bei den Ärzten). In beiden Gruppen wurde bei $10 \%$ der Patienten eine Angina tonsillaris und eine Bronchitis diagnostiziert, eine Sinusitis bei $10 \%$ in der KMT-Gruppe und $3 \%$ der Ärzte-Gruppe, eine Otitis bei $10 \%$ in der Ärzte-Gruppe und $3 \%$ in der KMT-Gruppe.

\section{Vergleich von Behandlungsqualität und Outcome}

Das KMT setzte signifikant häufiger NSAR ein als die Ärzte/-innen: $63 \%$ versus $20 \%$; Chi $=9,9 ; \mathrm{p}=0,002$.

Das KMT verordnete signifikant weniger Antibiotika als die Ärzte/-innen: $10 \%$ versus $43 \%$; $\mathrm{Chi}=6,9 ; \mathrm{p}=0,009$. In der KMT-Gruppe war der Outcome bei $97 \%$ der Patienten/-innen positiv, in der Ärztegruppe bei $83 \%$. Der Unterschied ist nicht signifikant: $\mathrm{Chi}=1,7 ; \mathrm{p}=0,197$. In der KMT-Gruppe wurde für neun Patienten/-innen eine elektive Nachkontrolle geplant. In acht Fällen war diese positiv, in einem Fall zeigte sich keine Besserung der Beschwerden. In der Ärzte-Gruppe erfolgte bei sieben Patienten/-innen eine geplante Nachkontrolle, alle Patienten/-innen wiesen eine Regredienz der Beschwerden auf (positiver Outcome). Fünf Patienten stellten sich wegen ausbleibender Besserung innert sieben Tagen erneut ungeplant vor. Abbildung 1 zeigt die Ergebnisse des Vergleichs von Behandlungsqualität und Outcome der Behandlung.

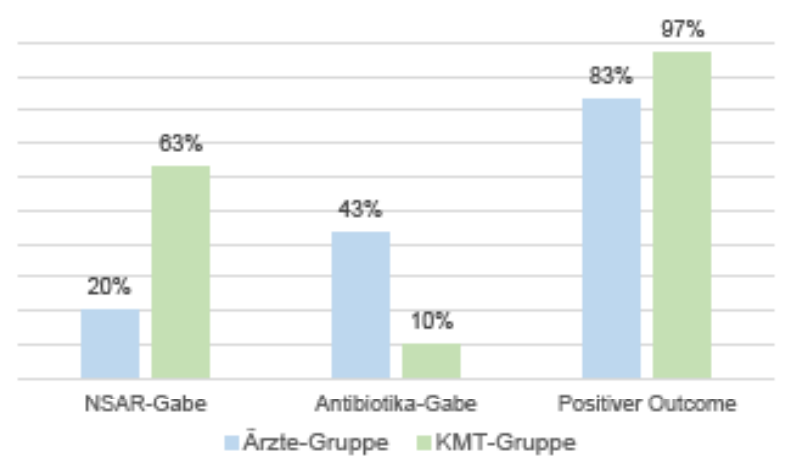

Abbildung 1: Vergleich von Behandlungsqualität und Outcome

\section{DISKUSSION}

In der durch das KMT behandelten Patienten/innengruppe war die Antibiotikaverschreibung signifikant geringer, ohne dass sich dadurch das Outcome verschlechtert hat. Die leicht erhöhten Entzündungswerte in der Ärzte-Gruppe können diesen Unterschied nicht 
erklären, auch in Betracht der häufigen Diagnose eines meist unspezifischen Infektes der Atemwege. Das Ergebnis bestätigt vielmehr die Beobachtung, dass Ärzte/-innen bei Infekten der oberen Atemwege entgegen aktueller Guidelines noch zu häufig Antibiotika einsetzen (Catho \& Huttner, 2018, S. 957), was zu einer Zunahme von Resistenzen in der Schweiz führt (Gasser et al., 2018, S. 943). Antibiotika haben jedoch bei Infekten der oberen Atemwege häufig mehr Nachteile als Vorteile und sollten daher nur unter strenger Indikationsstellung verordnet werden (Catho \& Huttner, 2018, S. 957). Für die Indikationsstellung gibt es mittlerweile für die Praxis sehr gut anwendbare Guidelines der Schweizerischen Fachgesellschaft für Infektiologie (SGINF, 2020). In der Top-5-Liste der Kampagne Smarter Medicine rät die Gesellschaft zudem, Antibiotika bei Infektionen der oberen Atemwege nur zurückhaltend einzusetzen (smartermedicine, 2020). Im KMT-Handbuch sind diese Guidelines integriert und auf die Umsetzung wird durch die ärztliche Supervision geachtet.

Der meist positive Outcome zeigt, dass bei gut standardisierten Behandlungsfällen der Einsatz von APN und $\mathrm{CN}$ in der hausärztlichen Praxis möglich ist und eventuell sogar die Behandlungsqualität verbessert. Das Ergebnis deckt sich mit internationalen Studien, bei denen bereits gezeigt wurde, dass der Einsatz dieser Berufsgruppe in der Grundversorgung zu einer Verbesserung der Qualität in der medizinischen Behandlung führen kann (Laurant et al., 2018, S. 2).

Die Interprofessionalität wird durch die Integration dieser Rolle gefördert, da ein neues Bindeglied zwischen Ärzten und MPAs entsteht. Nach den Erfahrungen der Gruppenpraxis Medbase Winterthur Neuwiesen wird durch den häufigen fachlichen Austausch auch das Teambuilding einer Praxisgemeinschaft gefördert. Grundlage für das Funktionieren dieser Zusammenarbeit sind eine ständige und gute Kommunikation sowie gegenseitige Wertschätzung und Vertrauen. Diese lassen sich empirisch jedoch nur schwer erfassen und können lediglich als Erfahrungen berichtet werden. Die Zufriedenheit der Patienten/-innen wurde bisher nicht gezielt erfasst; diese könnte mittels einer Fragebogenstudie erhoben werden. Negative Rückmeldungen/Reklamationen nach einer Behandlung durch das KMT seitens der Patienten/-innen gab es bisher keine. Dagegen gab es häufig positive Rückmeldungen. Wichtig ist beim Einsatz des KMT, die Transparenz bezüglich der nicht-ärztlichen Funktion von APN und $\mathrm{CN}$ gegenüber den Patientinnen und Patienten. Durch die längere Konsultationszeit von 30 Minuten beim KMT im Vergleich zu 15 Minuten bei den Ärzten/-innen bestand hier eher die Möglichkeit, individuell auf die
Beschwerden einzugehen und zu erklären, warum zum Beispiel kein Antibiotikum zum Einsatz kam. Dies kann dazu beitragen, die Compliance und möglicherweise auch das Outcome bei diesen Patientinnen und Patienten $\mathrm{zu}$ verbessern. Die Fokussierung auf bestimmte Krankheitsbilder führte insgesamt zu einer guten Strukturierung der Konsultation, sodass regelmäßig die Vitalwerte der Patientinnen und Patienten erfasst wurden. Durch die gemessenen Blutdruckwerte wurde nicht nur der Allgemeinzustand weiter objektiviert, sondern auch die Möglichkeit gegeben, nicht erkannte Hypertonien entdecken zu können. Grundsätzlich ist es wichtig zu betonen, dass für die Umsetzung geeignetes Personal und eine entsprechende Praxisorganisation notwendig sind.

\section{Einschränkungen}

Diese Studie basiert auf Daten von 60 Patienten/-innen mit Symptomatik einer einfachen Atemweginfektion. Für ein umfassenderes Bild und allgemeingültige Aussagen müsste die Behandlung von mehr Patienten/-innen mit einem breiteren Spektrum an Symptomen untersucht werden. Die interprofessionelle Zusammenarbeit lässt sich durch die Teamzusammensetzung objektivieren, die Interaktionen und die Auswirkungen auf das Team können bisher nur als subjektive Erfahrung berichtet werden. Hierzu bedarf es objektivierender Untersuchungsmethoden.

\section{Fazit}

Die retrospektive Analyse der Patientendaten bestätigt Erfahrungen in der Gruppenpraxis Medbase Winterthur Neuwiesen, dass sich die Rolle der APN/CN gut für die Behandlung von definierten Behandlungsfällen im hausärztlichen Bereich eignet und untermauert internationale Studien zu diesem Thema (Laurant et al., 2018, S. 2). Die Integration dieser Berufsgruppe kann insgesamt zu einem Gewinn im hausärztlichen Setting führen. Als häufiges Argument für dessen Einsatz findet sich die zunehmende Knappheit ärztlicher und ökonomischer Ressourcen (Künzi \& Detzel, 2007, S. 60). Neben der Entlastung der Ärzte/-innen und einer Kostensenkung ergaben sich noch weitere Vorteile. $\mathrm{Zu}$ den Benefits zählt eine Stärkung der Interprofessionalität, ein Wissenstransfer von APN/CN zu den MPA und als Folge eine Verbesserung der Behandlungsqualität und des Teamgeists. Eine interprofessionelle Zusammenarbeit im ambulanten Gesundheitssektor durch die Integration von APN/CN zahlt sich somit für Patientinnen und Patienten und die beteiligten Fachkräfte aus. Die Politik ist gefragt, die entsprechenden Rahmenbedingungen zu schaffen. 


\section{Ethische Prüfung, Registrierung}

Diese Studie wurde im Rahmen der internen Qualitätssicherung der Gruppenpraxis Medbase Winterthur Neuwiesen durchgeführt. Gemäß Richtlinien der Kantonalen Ethikkommission Zürich (KEK) ist dafür keine Begutachtung/Genehmigung erforderlich (https:// www.zh.ch/de/gesundheit/ethik-humanforschung/ zustaendigkeit-kantonale-ethikkommission.html). In einer telefonischen Anfrage wurde dies von der KEK bestätigt.

\section{Interessenkonflikte}

Die Autoren/-innen arbeiten alle in der MedbaseGruppenpraxis Neuwiesen Winterthur. Die Studie wurde im Rahmen der internen Qualitätssicherung mit Mitteln der Gruppenpraxis durchgeführt. Es bestehen keine Interessenkonflikte.

\section{Literaturverzeichnis}

BundesamtfürGesundheit(BAG).FörderprogrammInterprofessionalität im Gesundheitswesen 2017-2020. Zugriff am 12.09.2020. https:// www.bag.admin.ch/bag/de/home/strategie-und-politik/nationalegesundheitspolitik/foerderprogramme-der-fachkraefteinitiativeplus/foerderprogramme-interprofessionalitaet.html

Catho, G. \& Huttner, B. (2018). Best practice in der Antibiotikatherapie. Swiss Medical Forum - Schweizerisches Medizin-Forum (46). EMH Media. Zugriff am 12.09.2020. https://medicalforum.ch/ article/doi/smf.2018.03389

Gasser, M., Schrenzel, J. \& Kronenberg, A. Aktuelle Entwicklung der Antibiotikaresistenzen in der Schweiz. Swiss Medical Forum - Schweizerisches Medizin-Forum (46). EMH Media. Zugriff am 12.09.2020. https://medicalforum.ch/article/doi/smf.2018.03404

Konferenz der kantonalen Gesundheitsdirektorinnen und -direktoren. Integrierte Versorgung in den Kantonen. Praxisbeispiele für integrierte Versorgung. Zugriff am 24.09.2020. https://www.gdkcds.ch/de/gesundheitsversorgung/integrierte-versorgung

Künzi, K. \& Detzel, P. (2007). Innovationen in der ambulanten Grundversorgung durch vermehrten Einbezug nichtärztlicher Berufsleute. Obsan. Zugriff am 12.09.2020. https://www.obsan. admin.ch/sites/default/files/publications/2015/ad27_nichtaerztl_ nurd.pdf

Laurant, M., Van der Biezen, M., Wijers, N., Watananirun, K., Kontopantelis, E. \& Van Vught, AJAH. (2018). Nurses as substitutes for doctors in primary care. Cochrane Database. Zugriff am 12.09.2020. https://www.cochranelibrary.com/cdsr/ doi/10.1002/14651858.CD001271.pub3/epdf/abstract

Maier, C., Aiken, L. \& Busse, R. (2017). Nurses in advanced roles im primary care: Policy levers for implementation. Health Working Papers (98). OECD Publishing. Zugriff am 12.09.2020. https:// www.oecd-ilibrary.org/social-issues-migration-health/nurses-inadvanced-roles-in-primary-care_a8756593-en

Schweizerische Gesellschaft für Infektiologie, SGINF. (2020). Smarter Medicine Guidelines. Zugriff am 30.09.2020. https://www.sginf. ch/guidelines/guidelines-overview.html smarter medicine (2020). Top 5 Liste Infektiologie. Zugriff am 30.9.2020. https://www.smartermedicine.ch/de/top-5-listen/infektiologie.html

Zürcher Hochschule für Angewandte Wissenschaften ZHAW (2020). Symposien zu Advanced Practice Nursing. Zugriff am 30.09.2020. https://www.zhaw.ch/de/gesundheit/institute-zentren/ipf/ tagungen-symposien/advanced-practice-nursing-apn/ 